\title{
A Importância das Órteses de Membros Inferiores na Distrofia Muscular de Duchenne - Revisáo
}

\author{
The Importance of Orthosis for the Lower Limbs In Duchenne Muscular Dystrophy \\ Naelka dos Anjos Fernandes', Denise Caldeira Troise', Francis Meire \\ Fávero $^{3}$, Sissy Veloso Fontes ${ }^{4}$, Acary Souza Bulle Oliveira
}

\section{RESUMO}

Introduçáo. A habilidade motora do paciente com Distrofia Muscular de Duchenne decresce progressivamente e a fisioterapia é fundamental no tratamento destes pacientes para manutenção da força muscular e amplitude de movimento, por meio de alongamentos diários e ortetização. Objetivo. Identificar a importância das principais órteses de membros inferiores utilizadas no tratamento de pacientes com Distrofia Muscular de Duchenne. Método. Foram realizadas buscas eletrônicas nas bases de dados MEDLINE, LILACS, SCIELO a fim de identificar os artigos científicos indexados e publicados de 1979 a 2010. Resultados. Dez artigos foram selecionados. Dos artigos selecionados, um era estudo retrospectivo, oito eram estudos prospectivos, um era um estudo prospectivo randomizado. Os artigos tratavam dos tipos de órteses mais utilizadas no tratamento de pacientes com Distrofia Muscular de Duchenne, e dos benefícios que o uso desses dispositivos proporcionava aos pacientes. Conclusóes. A órtese mais utilizada é a Knee-Ankle-Foot (KAFO), a qual é importante para prevenção/minimização de contraturas e deformidades, prolongamento da marcha e ortostatismo dos pacientes.

Unitermos. Distrofia Muscular de Duchenne, Aparelho Ortopédico, Fisioterapia.

Citaçáo. Fernandes NA, Troise DC, Fávero FM, Fontes SV, Oliveira ASB. A Importância das Órteses de Membros Inferiores na Distrofia Muscular de Duchenne - Revisão.

\begin{abstract}
Introduction. The motor skills of patients with Duchenne Muscular Dystrophy decrease gradually and physical therapy is essential in treating these patients to maintain muscle strength and range of motion through stretching exercises daily and orthosis. Objective. To identify the importance of lower limb orthosis used for the treatment of patients with Duchenne muscular dystrophy. Method. We conducted electronic searches in MEDLINE, LILACS, and SCIELO to identify scientific articles published and indexed from 1979 to 2010 . Results. Ten articles were found. Out of the selected articles, one was a retrospective study, eight were prospective studies, and one was a prospective randomized study. The articles dealt with the most types of orthosis used for the treatment of patients with Duchenne muscular dystrophy, and the benefits that the use of these devices afforded to patients. Conclusions. The orthosis used more frequently is the KneeAnkle-Foot (KAFO), which is important for minimization of deformities and contractures, prolonged gait and patients orthostatism.
\end{abstract}

Keywords. Duchenne Muscular Dystrophy, Orthosis, Physical Therapy.

Citation. Fernandes NA, Troise DC, Fávero FM, Fontes SV, Oliveira ASB. The Importance of Orthosis for the Lower Limbs In Duchenne Muscular Dystrophy.

\footnotetext{
Trabalho realizado na Universidade Federal de Sáo Paulo - UNIFESP, Sáo Paulo-SP, Brasil.

1.Fisioterapeuta Especialista em Doenças Neuromusculares pela UNIFESP, São Paulo-SP, Brasil.

2.Fisioterapeuta Mestre em Ciências da Reabilitação pelo Departamento de Fonoaudiologia, Fisioterapia e Terapia Ocupacional da Faculdade de Medicina da USP, São Paulo-SP, Brasil.

3.Fisioterapeuta, Doutora em Ciências, pelo Departamento de Neurologia/ Neurocirurgia da UNIFESP, São Paulo-SP, Brasil.

4.Fisioterapeuta, Educadora Física, Doutora, Docente da UNIFESP e UMESP, São Paulo-SP, Brasil.

5.Neurologista, Doutor, Professor filiado da disciplina de Neurologia do Departamento de Neurologia e Neurocirurgia da UNIFESP, São Paulo-SP, Brasil.
}

Endereço para correspondência:

Denise Caldeira Troise

R. Celso de Azevedo Marques 475 apt. 41, Mooca CEP 03122010, São Paulo-SP, Brasil.

E-mail: denisetroise@hotmail.com 


\section{INTRODUÇÃO}

A Distrofia Muscular de Duchenne (DMD) é o tipo mais frequente dentre as distrofias musculares, afetando cerca de um em cada 3500 meninos nascidos vivos. Aproximadamente um terço dos casos são causados por novas mutaçôes, os outros são casos familiares, nestes a prevalência é menor, cerca de 1 em cada 18.000 indivíduos ${ }^{1}$.

Os indivíduos com DMD apresentam atraso no desenvolvimento motor, tais como dificuldade em levantar-se e ficar em pé, dificuldade na marcha e quedas frequentes. $\mathrm{O}$ acometimento motor é simétrico e proximal, ocorre inicialmente na cintura pélvica (do segundo ao quarto ano de vida) e por volta dos seis anos de idade a cintura escapular também é acometida ${ }^{2-4}$.

A fisioterapia é fundamental no tratamento destes pacientes para manutenção da força muscular e amplitude de movimento, os exercícios de alongamento diários dos músculos gastrocnêmio-sóleo, flexores de quadril e banda iliotibial combinados com a ortetização são muito bem sucedidos em controlar as contraturas de membros inferiores desses pacientes, e consequentemente a funcionalidade. Os principais objetivos da fisioterapia são manter ou melhorar a força muscular, maximizar a capacidade funcional e minimizar o desenvolvimento de contraturas através do uso de órteses. As órteses de membros inferiores fazem parte do tratamento destes pacientes ${ }^{5,6}$.

A utilização de órteses de membros inferiores é comumente instituída como uma maneira de prolongar a independência funcional do indivíduo com DMD, especialmente a partir do momento em que ocorre o decréscimo da deambulação devido à combinação da fraqueza da musculatura proximal de membros inferiores com a contratura do tendão de Aquiles ${ }^{7}$.

Com as recomendaçôes fisioterapêuticas adequadas (alongamentos, exercícios funcionais, ortetização), os pacientes com DMD poderão manter o posicionamento adequado dos membros inferiores, especialmente dos pés, podendo fazer uso de calçados sem dificuldade. A fisioterapia associada à cirurgia de correção e ortetização promove claro benefício ao paciente de DMD com a correção em longo prazo das contraturas das extremidades inferiores ${ }^{8}$.

\section{MÉTODO}

Revisão Tradicional da Literatura com análise crítica. Foram realizadas buscas eletrônicas nas bases de dados MEDLINE (Medlars Online), LILACS (Literatura Latino-Americana e do Caribe em Ciências da Saúde), SCIELO (The Scientific Electronic Library Online), a fim de identificar os artigos científicos indexados e publicados de 1979 a 2010. As estratégias de busca utilizadas foram "Duchenne Muscular Dystrophy” AND "Orthoses OR Ortheses OR Orthisis OR Splints OR Braces OR Ambulation" OR Rehabilitation" OR "Physical Therapy".

Como critérios de inclusão foram utilizados ensaios clínicos, estudos prospectivos e retrospectivos, que tratem acerca da intervenção das principais órteses de membros inferiores em crianças e adultos jovens com diagnóstico de DMD segundo critérios padronizados. Os critérios de exclusão adotados foram artigos que abordavam a utilização de órteses para membros superiores e tronco, revisóes de literatura, aplicação de questionários, opinião de especialista e artigos que tratavam do desenvolvimento de um tipo específico de dispositivo ortético e/ou abordagem sobre material para fabricação das órteses.

\section{RESULTADOS}

No total, foram identificados 35 artigos e após a análise do título, desenho do estudo, tipo de intervenção e desfecho dos mesmos, conforme critérios de inclusão, 10 artigos foram selecionados. Desses artigos selecionados, 1 era estudo retrospectivo, 8 eram estudos prospectivos, 1 era um estudo prospectivo randomizado. Segue abaixo a caracterização dos estudos (Quadro 1).

\section{DISCUSSÃO}

Nesta revisão tradicional da literatura com análise crítica, foram estudados por Bach em 1991, 13 meninos com diagnóstico de DMD segundo critérios já estabelecidos ${ }^{9}$. No pós-operatório todos os pacientes foram submetidos à intervenção fisioterapêutica e uso de órteses KAFO e LLB (Long Leg Brace) a partir do $2^{\circ}$ dia. Após o procedimento cirúrgico e o período de fisioterapia, observou-se que todos os pacientes foram capazes de prolongar o tempo de bipedestação não assistida e 11 pacientes apresentaram redução significativa do número de quedas 
Quadro 1

Descrição dos estudos

\begin{tabular}{c|c|c|c|}
\hline ID & Tipo de estudo & No de pacientes & Órtese utilizada \\
\hline VIGNOS et $\mathrm{al}^{5}, 1983$ & Prospectivo & 50 & KAFO \\
\hline Bach et $\mathrm{al}^{9}, 1991$ & Prospectivo & 13 & LLB e KAFO \\
\hline GRANATA et $\mathrm{al}^{10}, 1988$ & Prospectivo & 17 & Tutor Longo \\
\hline HECKMATT et $\mathrm{al}^{11}, 1985$ & Retrospectivo & 57 & KAFO \\
\hline HYDE et a ${ }^{12}, 1982$ & Prospectivo & 30 & KAFO \\
\hline SCOTT et $\mathrm{al}^{13}, 1981$ & Prospectivo & 59 & Não relatada \\
\hline VIGNOS et $\mathrm{al}^{14}, 1996$ & Prospectivo & 144 & KAFO \\
\hline WILLIAMS et $\mathrm{l}^{15}, 1984$ & Prospectivo & 69 & Náo relatada \\
\hline ZITER et $\mathrm{al}^{16}, 1979$ & Prospectivo & 17 & KAFO \\
\hline HYDE et a ${ }^{17}, 2000$ & Prospectivo & 27 & Não relatada \\
\hline
\end{tabular}

LLB: "Long Leg Brace"

KAFO: "Knee-Ankle-Foot-Orthose"

por mês de $84 \pm 87$ para $1 \pm 1(\mathrm{p}<0.05)$. Seis pacientes foram capazes de deambular por $2.48 \pm 1.03$ anos após a intervenção $(\mathrm{p}=0.034)$.

Já entre os anos de 1984 e 1987, um estudo com amostra de dezessete pacientes com DMD descreveu que os mesmos foram submetidos à cirurgia nos membros inferiores e consequentemente um programa de fisioterapia com a utilização de tutor longo no momento da perda da deambulação ${ }^{10}$. O tratamento pós-operatório compreendia a aplicação de gesso nos membros inferiores, com o qual após 5 dias as crianças começavam treino em bipedestação e após mais 2 dias iniciavam o treino de marcha. Após a cirurgia e a fisioterapia, todos os pacientes foram capazes de caminhar sem auxílio por cerca de 1 ano e 8 meses.

Entre 1977 e 1982, em uma outra pesquisa, cinquenta e sete meninos foram acompanhados durante 6 meses com o uso de órteses $\mathrm{KAFO}^{11}$. Após o primeiro dia pós-operatório os pacientes foram submetidos a treino em bipedestação para descarga de peso fazendo uso de gesso nos membros inferiores. No $3^{\circ}$ dia de pós-operatório o gesso foi retirado e colocada a órtese KAFO para início do treino de marcha. Os autores citam que a utilização de órteses KAFO ajuda a manter uma aparência mais agradável e ao utilizá-las os pacientes são capazes de levantar e caminhar com/sem auxílio, retardando o aparecimento de deformidades, em especial contraturas de quadril e joelho, pé equino e escoliose.
Assim, entre os anos de 1976 e 1980, outro estudo realizou a avaliação quantitativa seriada de 30 meninos com diagnóstico de $\mathrm{DMD}^{12}$. Todos os pacientes foram adaptados à órtese KAFO. Os autores demonstraram que os tipos de marcha independente e assistida no nível 6 da escala de Vignos continuou por cerca de 22.17 meses com um intervalo de 5 a 48 meses em 24 dos 30 meninos participantes do estudo. Com isso, sugeriram que a deambulação em pacientes com DMD pode ser prolongada com o uso de órteses, e o fator preditivo de maior importância para esta ocorrência é a presença de força muscular residual nos abdutores de quadril e extensores de joelho.

Em outra pesquisa, 59 meninos com diagnóstico de DMD fizeram parte do estudo ${ }^{13}$. Os resultados mostraram que ocorre um atraso na perda da dorsiflexão dos tornozelos e na contratura do tendáo calcâneo de meninos submetidos a alongamentos diários e uso de dispositivos ortéticos no período noturno durante os estágios iniciais da DMD.

As variáveis prognósticas mais importantes na duração da deambulação com órteses são: capacidade vital, porcentagem residual de força muscular e coeficiente de creatinina ${ }^{14}$. $\mathrm{O}$ mesmo autor em um estudo mais recente afirma que o controle do avanço das contraturas de membros inferiores por meio da utilização de órteses retarda o avanço da fraqueza muscular dos pacientes com DMD, os quais retém a habilidade de caminhar por mais tempo ${ }^{5,9}$. 
Um estudo com 69 meninos divididos em 3 grupos (grupo 1 - locomoção independente; grupo 2- locomoção por meio de órteses e grupo 3- cadeirantes), relatou que por meio de um programa de alongamentos passivos supervisionados por um fisioterapeuta, aliados ao uso de órteses durante o período noturno e cirurgias corretivas, prolongaram a marcha e a capacidade dos pacientes de ficarem de pé em torno de 20 meses $^{15}$.

A ausência de contraturas graves, prescrição ortética acurada e a cooperação do paciente e da família para seguir as recomendaçóes são extremamente importantes para alcançar os objetivos propostos ${ }^{16}$.

Esta revisão mostrou que o uso da Knee-AnkleFoot-Orthose (KAFO) associado às cirurgias corretivas é de fundamental importância para a prevenção/minimização de contraturas e deformidades, assim como no prolongamento da marcha e do ortostatismo desses pacientes e um intenso trabalho da Fisioterapia é um recurso complementar no tratamento de pacientes com DMD, entretanto poucos estudos relataram os efeitos da AFO na prevenção de contraturas e deformidades de tornozelos.

\section{CONCLUSÃO}

Esta revisão tradicional da literatura sugere que a órtese de membro inferior mais utilizada em pacientes de DMD nos artigos analisados foi a Knee-Ankle-Foot (KAFO). Os estudos avaliados mostraram que a utilização desse tipo de órtese é de fundamental importância para a prevenção/minimização de contraturas e deformidades, assim como no prolongamento da marcha e do ortostatismo desses pacientes e os artigos pesquisados mostraram que as cirurgias corretivas associadas à intervenção fisioterapêutica e o uso correto de órteses são fatores que contribuem positivamente para o tratamento de pacientes com distrofia muscular de Duchenne. Entretanto, nenhum artigo analisou o uso da órtese sem estar associada a correçóes cirúrgicas.

\section{REFERÊNCIAS}

1.Dooley J, Gordon KE, Dodds L, MacSween J. Duchenne Muscular Dystrophy: A 30-Year Population-Based Incidence Study. Clin Pediatr (Phila)
2010;49:177.

http://dx.doi.org/10.1177/0009922809347777

2.Bushby K, Finkel R, Birnkrant DJ, Case LE, Clemens PR, Cripe L, et al. Diagnosis and management of Duchenne Muscular Dystrophy, part 1: diagnosis and pharmacological and psychosocial management. Lancet Neurol 2010;9(1):77-93.

http://dx.doi.org/10.1016/S1474-4422(09)70271-6

3.Silva CT, Fonseca DJ, Mateus H, Contreras N, Restrepo CM, Bogotá DC. Duchenne and Becker's muscle dystrophy: A molecular vision. Acta Méd Colomb 2005;30:112-16.

4.Parreira SLS, Resende MBD, Peduto MDC, Nagahashi MSK, Carvalho MS, Reed UC. Quantification of muscle strength and motor ability in patients with Duchenne muscular dystrophy on steroid therapy. Arq. Neuropsiquiatr 2007;65(2A):245-50.

http://dx.doi.org/10.1590/S0004-282X2007000200011

5.Vignos PJ, Wagner MB, Kaplan JS, Spencer GE. Predicting the success of reabulation in patients with Duchenne Muscular Dystrophy. J Bone Jt. Surg. (Am) 1983;65(6):719-28.

6.Eagle M. Report on the Muscular Dystrophy Campaign workshop: Exercise in neuromuscular diseases Newcastle, January 2002. Neuromuscular Disorders 2002;12:975-983.

http://dx.doi.org/10.1016/S0960-8966(02)00136-0

7.Garralda ME, Muntoni F, Cunniff A, Caneja AD. Knee-Ankle-Foot orthosis in children with Duchenne Muscular Dystrophy: user views and adjustment. Eur J. Paediatr Neurol. 2006;10:186-191.

http://dx.doi.org/10.1016/j.ejpn.2006.07.002

8.Scher DM, Mubarak S. Surgical Prevention of Foot Deformity in Patients With Duchenne Muscular Dystrophy. J Pediatr Orthop 2002;22(3):384-91. http://dx.doi.org/10.1097/00004694-200205000-00024

http://dx.doi.org/10.1097/01241398-200205000-00024

9.Bach JR, McKeon J. Orthopedic surgery and rehabilitation for the prolongation of brace-free ambulation of patients with Duchenne Muscular Dystrophy. Am J Phys Med Rehab. 1991;70:323-9.

10.Granata C, Giannini S, Rubbini L, Corbascio M, Bonfiglioli S, Sabattini L, et al. Orthopedic surgery to prolong walking in Duchenne Muscular Dystrophy. Chir Organi Mov Arch. 1988;73(3):237-48.

11.Heckmatt JZ, Dubowitz V, Hyde SA, Florence J, Gabain AC, Thompson N. Prolongation of walking in Duchenne Muscular Dystrophy with lightweight orthoses: reviem of 57 cases. Dev Med Child Neurol 1985;27(2):149-54. http://dx.doi.org/10.1111/j.1469-8749.1985.tb03763.x

12.Hyde SA, Scott OM, Goddard CM, Dubowitz V. Prolongation of ambulation in Duchenne Muscular Dystrophy by appropriate orthoses. Physiotherapy 1982;68(4):105-8.

13. Scott OM, Hyde SA, Goddard C, Dubowitz V. Prevention of deformity in Duchenne Muscular Dystrophy. Physiotherapy 1981;67(6):177-80.

14.Vignos PJ, Spencer GE, Archibald KC: Management of progressive muscular dystrophy of childhood. JAMA 1963;184:103-110.

http://dx.doi.org/10.1001/jama.1963.03700150043007

15.Williams EA, Read L, Ellis A, Morris P, Galasko CS. The management of equines deformity in Duchenne Muscular Dystrophy. J Bone Jt Surg (Am) 1984;66(4):546-50.

16.Ziter FA, Allsop KG. The value of orthoses for patients with Duchenne Muscular Dystrophy. Physical Therapy 1979;59(11):1361-65.

17.Hyde SA, Floytrop I, Glent S, Kroksmark AK, Salling B, Steffensen BF, et al. A randomized comparative study of two methods for controlling Tendo Achilles contracture in Duchenne Muscular Dystrophy. Neuromuscular Disorders 2000;10:257-63.

http://dx.doi.org/10.1016/S0960-8966(99)00135-2 